\title{
3 Research Square \\ EEG beta-band spectral entropy predicts the effects of drug treatment on patients with herpes zoster
}

\section{Mengying Wei}

Shenzhen University

\section{Yuliang Liao}

Department of Pain Medicine and Shenzhen Municipal Key Laboratory for Pain Medicine

Jia Liu

School of Biomedical Engineering, Health Science Center, Shenzhen University

\section{Linling Li}

School of Biomedical Engineering, Health Science Center, Shenzhen University

\section{Gan Huang}

School of Biomedical Engineering, Health Science Center, Shenzhen University

\section{Disen Li}

Department of Pain Medicine and Shenzhen Municipal Key Laboratory for Pain Medicine Jiabin Huang

Department of Pain Medicine and Shenzhen Municipal Key Laboratory for Pain Medicine Lizu Xiao

Department of Pain Medicine and Shenzhen Municipal Key Laboratory for Pain Medicine Zhiguo Zhang ( $\nabla$ zgzhang@szu.edu.cn )

\section{Research article}

Keywords: EEG; herpes zoster; power spectra entropy; machine learning; medication response

Posted Date: May 7th, 2019

DOl: https://doi.org/10.21203/rs.2.9483/v1

License: (a) (i) This work is licensed under a Creative Commons Attribution 4.0 International License. Read Full License 


\section{Abstract}

Background Medication is the main approach for early treatment of herpes zoster $(\mathrm{HZ})$, but it could be ineffective in some patients. It is highly desired to predict the medication responses in order to control the degree of pain for $\mathrm{HZ}$ patients. The present study is aimed to elucidate the relationship between medication outcome and neural activity using electroencephalography (EEG) and to establish a machine learning model for early prediction of the medication responses from EEG. Methods We acquired and analyzed eye-closed resting-state EEG data 1-2 days after medication from $70 \mathrm{HZ}$ patients with different drug treatment outcomes (measured 5-6 days after medicaiton): 45 medication-sensitive pain (MSP) patients and 25 medication-resistant pain (MRP) patients. EEG power spectral entropy (PSE) of each frequency band was compared at each channel between MSP and MRP patients, and those features showing sigificant difference between two groups were used to predict medication outcome with different machine learning methods. Results MSP patients showed significantly weaker beta-band PSE in the central-parietal regions than MRP patients. Based on these EEG PSE features and a k-nearest neighbors (k-NN) classifier, we can predicate the medication outcome with $80 \% \pm 11.7 \%$ accuracy, $82.5 \% \pm 14.7 \%$ sensitivity, $77.7 \% \pm 27.3 \%$ specificity and an AUC of 0.85 . Conclusion EEG beta-band PSE in the centralparietal region is predictive of the effectiveness of drug treatment on $\mathrm{HZ}$ patients, and it could potentially be used for early pain management and therapeutic prognosis.

\section{Background}

Herpes zoster $(\mathrm{HZ})$ is a rash that spreads in the skin with pain and blistering ${ }^{[1]}$. $\mathrm{HZ}$ is caused by the activation of the varicella zoster virus (VZV), which persists in the ganglion after primary infection. As the age increases and the immunosuppression decreases, VZV reactivates and travels along the sensory nerves to the skin, causing distinctive prodromal pain followed by eruption of the rash ${ }^{[1,2]}$. $\mathrm{HZ}$ is the most common neurological disorder in many countries, such as the USA, Canada and UK, and temporal increases in the incidence of $\mathrm{HZ}$ have also been reported ${ }^{[3-8]}$. $\mathrm{HZ}$ is often accompanied with psychological problems (such as anxiety and depression) and many serious complications, all of which seriously affect the patients' quality of the patients' life ${ }^{[9,10]}$.

Early intervention of $\mathrm{HZ}$ and $\mathrm{HZ}$-related complications, such as postherpetic neuralgia (PHN), is of great clinical importance, because the age is an important risk factor for developing $\mathrm{HZ}$ (i.e., the incidence of $\mathrm{HZ}$ and $\mathrm{HZ}$-related complications is increased with the age) ${ }^{[11]}$. To date, early intervention for $\mathrm{HZ}$ is generally achieved by medication, including analgesics and antivirals ${ }^{[1]}$. Drug treatment can decrease the duration and severity of the rash and can also relieve the pain associated with $\mathrm{HZ}$. Evidence has shown that antiviral therapy during the acute phase could effectively prevent PHN ${ }^{[12-14]}$. However, because of remarkable individual difference among patients, drug treatment is not always effective for each patient. In addition, the side-effects of some drugs may be serious when they are used in the long term. If the effect of drug treatment can be accurately predicted in an early stage, patients can get optimal and individualized drug treatment and non-responders can avoid staying on medications and the condition of 
pain for a prolonged period of time. Therefore, it is highly desired to precisely predict the outcome of drug treatment in order to achieve effective and precise treatments to control the degree of pain for $\mathrm{HZ}$ patients.

The effectiveness of medication on pain is generally evaluated as the degree of decrease in patients' subjective pain ratings after treatment. Although self-report is still the golden-standard for pain assessment in clinical practice, a variety of physiological correlates of pain have been found in past decades ${ }^{[15-17]}$. Particularly, a huge body of studies based on electroencephalography (EEG) have shown that pain perception is closely associated with certain types of brain rhythmical activities ${ }^{[18-20]}$. So, EEG has been widely used to study neuropathic pain and chronic pain ${ }^{[21-23]}$, with the aim to discover neural signatures of pain diseases for objective assessment of pain level or early diagnosis of these pain diseases. An early study showed that patients with severe forms of neurogenic pain exhibited higher EEG spectral power over the frequency range of $2-25 \mathrm{~Hz}{ }^{[18]}$. Another study showed that the average peak EEG alpha frequency of chronic pain patients was lowered than that of healthy controls ${ }^{[24]}$. By using magnetoencephalography (MEG), which is the measurement of the brain's magnetic field and is similar to EEG in terms of the signal characteristics and analysis methods, researchers found fibromyalgia (FM) patients had power increases in theta, beta and gamma bands, along with a slowing of the dominant alpha peak ${ }^{[25]}$. Recently, it has been reported that FM patients augmented theta activity in prefrontal and anterior cingulate cortices relative to healthy controls ${ }^{[21]}$.

However, most previous related studies used EEG to identify abnormal brain activity patterns of pain patients, but there are few studies concerning the use of EEG as a possible neural signature of drug treatment on pain. Actually, as revealed by many studies of other diseases, such as depression and epilepticus, EEG plays an important role as a neural correlate of effectiveness of drug treatment ${ }^{[26-28]}$. For example, a recent study explored EEG spectral power of patients with treatment-resistant depression, and found certain EEG features are correlated with the efficacy of ketamine treatment and could be used to predict drug effects ${ }^{[29]}$. These studies inspired us to search for EEG-based predicators of medications for $\mathrm{HZ}$ patients.

In the present study, we hypothesize that the medication effects on pain could be represented by EEG rhythmic features and aim to explore the relationship between EEG and drug treatment effects on $\mathrm{HZ}$. To this end, we recorded EEG activities of $\mathrm{HZ}$ patients 1-2 days after they took medication. To avoid uncomfortableness of patients and long operation time, we used a new dry-electrode EEG device instead of the traditional EEG devices that need conductive gel. Then, we compared EEG spectral features between responders and non-responders to identify treatment outcome-related EEG features. Further, based on the identified treatment outcome-related EEG features, we used various machine learning models to predict the effectiveness of drug treatment.

\section{Methods}




\section{Participants}

All patients were recruited from Department of Pain Medicine and Shenzhen Municipal Key Laboratory for Pain Medicine, the Affiliated Shenzhen Sixth Hospital of Guangdong Medical University, from January 2017 to July 2018. All patients have written informed consent and informed about experimental content. All study procedures were approved by Human Research Ethics Committee of the Affiliated Shenzhen Sixth Hospital of Guangdong Medical University.

The inclusion criteria for $\mathrm{HZ}$ patients were: (1) age ranged from 31 to 90 years; (2) the duration of $\mathrm{HZ}$ was less than 3 months. Exclusion criteria included: (1) had acute and chronic pain caused by other disease such as migraine headache, fibromyalgia; (2) had epilepsy or Alzheimer's disease that may affect the nervous system. All the patients were administrated the standardized medication protocol by experienced physicians for a week. These drugs included gabapentin, pregabalin, oxycodone/acetaminophen, tramadol and amitriptyline, and the dosage ranged from $75 \mathrm{mg}$ to $450 \mathrm{mg}$. The pain intensity was evaluated by using Visual Analogue Scale (VAS). For each patient, we recorded his/her VAS scores for three times: (1) $V_{A D}$ was measured when the patient was admitted to the hospital (1-2 days before medication), (2) $\mathrm{VAS}_{\mathrm{EEG}}$ was measured right before EEG acquisition 3 days after admission (1-2 days after medication), (3) VAS $_{\text {DIS }}$ was measured when the patient was discriminated as a medicationsensitive pain (MSP) patient (i.e., responder) or a medication-resistant pain (MRP) patient (nonresponder) one week after admission and 5-6 days after medication. A patient was defined as having MSP, if his/her VAS ${ }_{D I S}$ was less than half of VAS ${ }_{A D M}, V_{D I S}$ was less than 3. Otherwise, the patient was defined as having MRP. In addition, if the doctor thought the medication failed to improve the patient's quality of life, the patient was also defined as having MRP regardless of the pain ratings.

EEG data acquisition

EEG acquisition was performed in a quiet and bright room. Patients were seated in a comfortable chair and were asked to relax their muscles and close their eyes. We used a dry-electrode mobile wireless EEG system, Brain Product LiveAmp (Brain Product GmbH, Munich, Germany), to acquire EEG signals. This LiveAmp system was used because it can save operational time and reduce the uncomfortableness of patients. Six-minute resting-state EEG data were recorded using 32 scalp electrodes placed according to the International 10-20 system. The sampling rate was $500 \mathrm{~Hz}$ and electrode impedances were kept below $20 \mathrm{k} \Omega$.

\section{EEG data analysis}

The EEG data were preprocessed using Letswave (https://github.com/NOCIONS/letswave7), a free EEG signal-processing toolbox under the MATLAB (version R2017a, MathWorks) environment. First, apparent contaminations were manually removed by visual inspection. Then, data at bad channels were interpolated from data at adjacent channels. Next, EEG signals were re-referenced to a common reference, which is the average of EEG signals at all electrodes ${ }^{[30]}$. Continuous EEG data were then bandpass 
filtered between 0.1 and $30 \mathrm{~Hz}$. EEG data contaminated by eye-blinks and muscle artifacts were corrected using independent component analysis ${ }^{[31-33]}$. Independent components corresponding to eye blinks and muscle artifacts were rejected manually.

After preprocessing, clean EEG data were transformed into the frequency domain by the Welch's method (with 1024-point FFT, 50\% overlapping, and Hamming windows). Then we computed the power spectral entropy (PSE) ${ }^{[34]}$, which is the information entropy of power spectrum, at four frequency bands: delta $(0.5-4 \mathrm{~Hz})$, theta $(4-8 \mathrm{~Hz})$, alpha $(8-12 \mathrm{~Hz})$, and beta $(12.5-30 \mathrm{~Hz})$. PSE is a commonly used EEG feature and is calculated as, where $n$ is the number of frequency points within a frequency band of interest and is the EEG power at the $i$ th $(i=1,2, \ldots, n)$ frequency point. PSE quantifies the amount of information in the power spectrum of a given signal, and it can provide a more complete description about the signal's spectral properties than some other spectral features, such as absolute power, relative power, and peak frequency.

Next, we compared PSE of four frequency bands (theta, delta, alpha and beta) at each channel between MSP and MRP patients. PSE values that are significantly different between MSP and MRP patients within specific frequency bands and at specific channels were used as features to predict drug effects in a machine learning model.

\section{Results}

Demographic and behavioral results

Eighty HZ patients were recruited in this study, and ten of them were discarded because their EEG signals were heavily contaminated. Remaining $70 \mathrm{HZ}$ patients were grouped according to drug treatments: 45 were MSP and 25 were MRP. The demographic and clinical characteristics of all 70 patients are presented in Table 1, which listed the age, gender, education and clinical profiles (disease duration, VAS scores, and dosage of drugs). The two $\mathrm{HZ}$ groups did not differ significantly in gender, age and education. MRP patients showed significantly higher illness duration than MSP patients, and thus duration was regressed out in all group-level analyses. There was no significant difference of VAS scores in different periods between MSP and MRP patients.

\section{Comparison of EEG between MSP and MRP}

Controlling for age, gender and education, we compared PSE within different frequency bands at all channels between MSP and MRP patients using two-sample rank sum test. As shown in Figure 1, we found significant group differences over electrodes of central-parietal regions (including CP1, CP2 and $\mathrm{Pz}$ ) in the beta band. No differences were observed in the PSE within other frequency bands between MSP and MRP patients.

The mean and SD of PSE values at CP1, CP2 and Pz in the beta band were illustrated in Figure 2 . 
The classification performances of four prediction models, LDA, k-NN and SVM, and RF, are summarized in Table 2. The best performance was achieved by k-NN, which had $80 \% \pm 11.7 \%$ accuracy, $82.5 \% \pm 14.7 \%$ sensitivity, $77.7 \% \pm 27.3 \%$ specificity and an AUC of 0.85 . Figure 3 shows the Receiver Operating

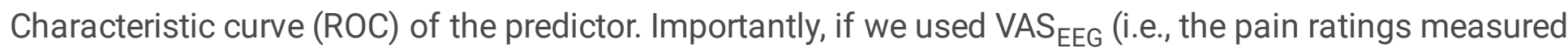
right before EEG acquisition) to predict the medication effects (with the criteria that, if a patient's VAS $S_{\text {EEG }}$ was less than half of VAS ADM $_{\text {or }}$ VAS $_{\text {EEG }}$ was less than 3, the patient was defined as MSP), we got the results: accuracy $=58.6 \%$, sensitivity $=69.1 \%$, and specificity $=42.9 \%$. By comparing the prediction results using EEG PSE features and pain ratings, which were acquired at the same day, we can see that PSE features can achieve much higher accuracy, sensitivity, and specificity than pain ratings.

\section{Discussion}

In this study, we compared EEG power feature (PSE) in HZ patients with different effects of drug treatment. We observed that the beta-band PSE at the central-parietal region was significantly different between MSP and MRP patients, implying that EEG beta rhythm could be a possible neural correlate of effective drug treatment. More importantly, our study used machine learning models to classify MSP and MRP patients with $80 \% \pm 11.7 \%$ accuracy, $82.5 \% \pm 14.7 \%$ sensitivity, $77.7 \% \pm 27.3 \%$ specificity and 0.85 AUC, suggesting EEG has the potential to predict the drug treatment effect on $\mathrm{HZ}$ patients in an early stage.

EEG as a Signature of Pain Treatment

EEG has been widely used in clinical pain research to find out EEG activity pattern of subjective pain experience. For example, some studies have shown that pain patients displayed enhanced power spectra at frontal and parieto-occipital regions $[22,24,36]$. According to these results, EEG spectral power could be used for diagnosis of pain disease and for monitoring the postoperative development of patients. The present study is different from previous EEG-based clinical pain studies in two aspects. First, this study is aimed to reveal EEG features that are related to different medication effects on $\mathrm{HZ}$-associated pain, not to identify EEG patterns that are different between pain patients and healthy people, patients of different pain diseases, or patients at different stage of a pain disease. Second, we proposed to used PSE, instead of the conventional power spectrum, as EEG features ${ }^{[37]}$. Literature has shown that the entropy-type parameters have a better ability to identify the complexity of EEG than spectral power ${ }^{[34,38]}$. For example, some studies reported that PSE was a sensitive parameter for EEG of imaginary hand movements, and was an effective index of focal ischemic cerebral injure ${ }^{[34,39]}$. We actually also compared EEG power and other spectral features between two MSP and MRP patients but did not find any difference.

Despite the fact that medication is the most common intervention for many pain-related diseases, including $\mathrm{HZ}$, our knowledge of medication effects on pain and on brain activity remains limited. Many EEG studies were aimed to identify the relationship between EEG and clinical outcomes ${ }^{[41,42]}$, and they 
indicated that the pre- or post-treatment EEG changes could be used to evaluate drug treatment outcomes. For example, some studies have demonstrated that drugs, such as pregabalin, influenced EEG characteristics ${ }^{[43,44]}$, suggesting that the changes of EEG activity are predictive of the drug effectiveness. A study explored the influence of gabapentin on healthy people's EEG activity showed that the peak frequency of alpha rhythm in the posterior region significantly decreases between healthy volunteer ${ }^{[45]}$. More recently, a paper reviewed EEG findings in analgesics and found that opioids could influence deltaband activity ${ }^{[46]}$. These studies suggested that EEG could reflect the overall altered neural activity including depression of behavior and mood in patients, which may predict the clinical outcome of drug treatment.

Our study found that, MSP patients showed significantly lower PSE in the beta band than MRP patients, which implies that if medication is effctive, the information (or the disorder/randomness) of the brain activity in the beta-band is suppressed. In another word, medication can restrain the beta-band EEG activity, if it is effective. This result is partly consistent with several similar studies which observed high frequency beta-band oscillations associated with pain processing ${ }^{[18,20,23,25]}$. An experimental pain study also showed that beta-band EEG activity in frontal/parietal regions is related to conscious somatic perception ${ }^{[40]}$. The results of our study implied that beta oscillatory activity in central-parietal regions may have an important role in the perception and processing of pain.

\section{Using EEG to Predict Drug Effectiveness}

Early prediction of drug treatment would aid in reducing the risk of side effects from inefficient treatment for patients. However, there is no study concerning the use of EEG as a predictor to drug treatment on pain diseases. According to our results, EEG features recorded several days before discrimination showed different patterns between MSP and MRP patients. So, the significant different features between MSP and MRP patients can be treated as characteristic inputs to build a classification (prediction) model to distinguish the effects of drug treatment. Our study provided a classification model with $80 \% \pm 11.7 \%$ accuracy, $82.5 \% \pm 14.7 \%$ sensitivity, $77.7 \% \pm 27.3 \%$ specificity and 0.85 AUC. This EEG prediction model may help doctors decide whether drug treatment is useful or not for $\mathrm{HZ}$ patients in an earlier stage.

It is important to mention that, EEG data we used in the study were recorded 1-2 days after drug treatment and 5-6 days before discrimination into MSP and MRP patients. By using pain ratings measured right before EEG acquisition, it was also possible to predict the medication responses. But our results in Table 2 showed that, using pain ratings $\mathrm{VAS}_{\mathrm{EEG}}$ (which was measured 1-2 days after medication and 5-6 days before discrimination) to predict medication response, the accuracy, sensitivity, and specificity were all lower than the prediction results from EEG features. The possible reason is that, pain ratings were subjective and could be biased by placebo effects in medication. Hence, the EEG PSE feature we found in the study may be more objective, making it suitable for building a prediction model.

Wireless Dry-electrode EEG 
In clinical practice, the acquisition of EEG is normally not convenient because it needs a long preparation time (for example, to inject conductive gel into electrodes) and the whole system (including the amplifier, cables, and computers) is cumbersome. Therefore, in this study we used a new wireless and dry-electrode EEG system, LiveAmp 32-channel (Brain Product GmbH, Munich, Germany) to acquire patients' EEG data. The wireless and portable EEG device with dry-contact sensors could significantly reduce the time of preparatory work. We compared the preparation time of the wireless dry-electrode system and a conventional wet-electrode EEG system, BrainAmp 32-channel (Brain Product GmbH, Munich, Germany), on 3 normal subjects and found the preparation time of LiveAmp is around 15-20 minutes while the average preparation time of BrainAmp is around 30-50 minutes. Importantly, patients did not report any feeling of uncomfortableness when wearing the wireless dry-electrode cap. Recently, a study systematically compared the wet-electrode wired EEG device and dry-electrode wireless EEG device in many aspects, and the results indicated that dry-electrode EEG device can effectively record EEG for research and clinical purposes ${ }^{[47]}$. So, dry-electrode wireless EEG devices are a promising tool for the EEG experiment on $\mathrm{HZ}$ patients.

\section{Limitations and Future Work}

The present study still has some limitations. First, the $\mathrm{HZ}$ patients took different medications during hospitalization. Different medications interaction or the use of a single medication may affect the EEG changes, which is a confounding factor of our study. Second, the repeatability and reliability of the identified EEG correlates of drug effectiveness should be validated using repeatedly measured data from the same cohort and multi-center data from different cohorts. Third, the neural mechanism underlying our finding remains unclear. It may be necessary to record multimodality data (such as MRI, fMRI, biochemical and genomic data) on a large sample to do a more comprehensive investigation of the neural mechanism of drug effectiveness on $\mathrm{HZ}$ patients. Finally, the sensitivity and specificity of this predict model are still not sufficient for clinical diagnosis, and we need to further improve the technique and to validate the results in a rigorous way.

\section{Conclusions}

In conclusion, the present study found that beta-band EEG power entropy at central-parietal region is correlated with the effectiveness of drug treatment on $\mathrm{HZ}$ patients and this EEG feature could potentially be used for early prediction of the drug effectiveness on $\mathrm{HZ}$ patients.

\section{Declarations}

Ethics approval and consent to participate

All patients have written informed consent and informed about experimental content. All study procedures were approved by Human Research Ethics Committee of the Affiliated Shenzhen Sixth Hospital of Guangdong Medical University. 
Consent for publication

Not applicable.

Availability of data and material

The data that support the findings of this study are available from the corresponding author upon reasonable request.

Competing interests

The authors declare that they have no competing interests.

Author's contributions

$\mathrm{YL}, \mathrm{JH}$ and $\mathrm{LL}$ designed the study and wrote the protocol. JL and MW performed data collection and management. MW performed the data analysis. MW wrote the first draft of the manuscript and critical revisions were contributed by ZZ and LX. All authors contributed to and have approved the final manuscript.

Abbreviations

EEG, electroencephalography; $\mathrm{HZ}$, herpes zoster; VZV, varicella zoster virus; $\mathrm{PHN}$, postherpetic neuralgia; MEG, magnetoencephalography; FM, fibromyalgia; VAS, visual analogue scale; MSP, medication-sensitive pain; MRP, medication-resistant pain; PSE, power spectral entropy; LDA, linear discriminant analysis; SVM, support vector machine; RF, random forest; $k-N N$, k-nearest neighbors; ROC, receive operating characteristic curve; $A U C$, the area under the receive operating characteristic curve; FDR, false discovery rate.

Acknowledgments

This study was funded by National Natural Science Foundation of China (Grant No. 81871443) and the Science, Technology and Innovation Commission of Shenzhen Municipality Technology Fund (Grant No. JCYJ20170818093322718). The funders had no role in study design, data collection and analysis, decision to publish, or preparation of the manuscript.

\section{References}

1. Sampathkumar P, Drage LA, Martin DP. Herpes zoster (shingles) and postherpetic neuralgia. Mayo Clin Proc. 2009;84(3):274-280.

2. Cohen JI. Clinical practice: Herpes zoster. N Engl J Med. 2013;369(3):255-63.

3. Donahue JG, Choo PW, Manson JE, Platt R. The incidence of herpes zoster. Arch Intern Med. 1995;155(15):1605-1609. 
4. Galil K, Choo PW, Donahue JG, Platt R. The sequelae of herpes zoster. Arch Intern Med. 1997;157(11):1209-1213.

5. Leung J, Harpaz R, Molinari NA, Jumaan A, Zhou F. Herpes zoster incidence among insured persons in the United States, 1993-2006: evaluation of impact of varicella vaccination. Clin Infect Dis. 2011;52(3):332-340.

6. Hales CM, Harpaz R, Joesoef MR, Bialek SR. Examination of links between herpes zoster incidence and childhood varicella vaccination. Ann Intern Med. 2013;159(11):739-745.

7. Wu PY, Wu HD, Chou TC, Sung FC. Varicella vaccination alters the chronological trends of herpes zoster and varicella. PLoS One. 2013;8(10): e77709.

8. Kawai K, Gebremeskel BG, Acosta CJ. Systematic review of incidence and complications of herpes zoster: towards a global perspective. BMJ Open. 2014;4(6): e004833.

9. Breuer J, Pacou M, Gautier A, Brown MM. Herpes zoster as a risk factor for stroke and TIA: a retrospective cohort study in the UK. Neurology. 2018;83(2): e27-33.

10. Lukas K, Edte A, Bertrand I. The impact of herpes zoster and post-herpetic neuralgia on quality of life: patient-reported outcomes in six European countries. Z Gesundh Wiss. 2012;20(4) :441-451.

11. Reynolds MA, Chaves SS, Harpaz R, Lopez AS, Seward JF. The impact of the varicella vaccination program on herpes zoster epidemiology in the United States: a review. J Infect Dis. 2008;197 Suppl 2: S224-227.

12. Gan EY, Tian EA, Tey HL. Management of herpes zoster and post-herpetic neuralgia. Am J Clin Dermatol. 2013;14(2): 77-85.

13. Chen N, Li Q, Yang J, Zhou M, Zhou D, He L. Antiviral treatment for preventing postherpetic neuralgia. Cochrane Database Syst Rev. 2014(2): CD006866.

14. Crosbie, B, Lucey, S, Tilson, L, Domegan L, Kieran J. Acute herpes zoster and post herpetic neuralgia in primary care: a study of diagnosis, treatment and cost. Eur J Clin Microbiol Infect Dis. 2018(37):627.

15. Chu Y, Zhao X, Han J, Su Y. Physiological Signal-Based Method for Measurement of Pain Intensity. Front Neurosci. 2017(11):279.

16. Jiang M, Mieronkoski R, Syrjälä E, et al. Acute pain intensity monitoring with the classification of multiple physiological parameters. J Clin Monit Comput. 2018 Jun 26.

17. Vuckovic A, Gallardo VJF, Jarjees M, Fraser M, Purcell M. Prediction of central neuropathic pain in spinal cord injury based on EEG classifier. Clin Neurophysiol. 2018;129(8):1605-1617.

18. Sarnthein J, Stern J, Aufenberg C, Rousson V, Jeanmonod D. Increased EEG power and slowed dominant frequency in patients with neurogenic pain. Brain. 2006;129(Pt 1):55-64.

19. Llinás RR, Ribary U, Jeanmonod D, Kronberg E, Mitra PP. Thalamocortical dysrhythmia: A neurological and neuropsychiatric syndrome characterized by magnetoencephalography. Proc Natl Acad Sci USA. 1999;96(26):15222-15227. 
20. Olesen SS, Hansen TM, Graversen C, Steimle K, Wilder-Smith OH, Drewes AM. Slowed EEG rhythmicity in patients with chronic pancreatitis: evidence of abnormal cerebral pain processing? Eur $J$ of Gastroenterol Hepatol. 2011;23(5):418-424.

21. Fallon N, Chiu Y, Nurmikko T, Stancak A. Altered theta oscillations in resting EEG of fibromyalgia syndrome patients. Eur J Pain. 2018;22(1):49-57.

22. Jensen MP, Sherlin LH, Gertz KJ, Braden AL, Kupper A, Gianas A, Howe JD, Hakimian S. Brain EEG activity correlates of chronic pain in persons with spinal cord injury: clinical implications. Spinal Cord. 2013;51(1):55-58.

23. Boord P, Siddall PJ, Tran Y, Herbert D, Middleton J, Craig A. Electroencephalographic slowing and reduced reactivity in neuropathic pain following spinal cord injury. Spinal Cord. 2008;46(2):118-123.

24. de Vries $\mathrm{M}$, Wilder-Smith $\mathrm{OH}$, Jongsma $\mathrm{ML}$, et al. Altered resting state EEG in chronic pancreatitis patients: toward a marker for chronic pain. J Pain Res. 2016(6):815-824.

25. Lim M, Kim JS, Kim DJ, Chung CK. Increased low- and high-frequency oscillatory activity in the prefrontal cortex of fibromyalgia patients. Font Hum Neurosci. 2016(10):111.

26. Basha MM, Alqallaf A, Shah AK. Drug-induced EEG pattern predicts effectiveness of ketamine in treating refractory status epilepticus. Epilepsia. 2015;56(4): e44-48.

27. Tian Y, Yang L, Xu, W, e al. Predictors for drug effects with brain disease: Shed new light from EEG parameters to brain connectomics. Eur J Pharm Sci. 2017(110):26-36.

28. Colic S, Wither RG, Lang M, Zhang L, Eubanks JH, Bardakjian BL. Prediction of antiepileptic drug treatment outcomes using machine learning. J Neural Eng. 2017;14(1): 016002.

29. Cao Z, Lin C, Ding W, Chen M, Li C, Su T. Identifying Ketamine Responses in Treatment-Resistant Depression Using a Wearable Forehead EEG. IEEE Trans Biomed Eng, 2018; doi: 10.1109/TBME.2018.2877651.

30. Dien J. Issues in the application of the average reference: review, critiques, and recommendations. Behav Res Methods Instrum Comput. 1998;30(1):34-43.

31. Makeig S, Jung TP, Bell AJ, Ghahremani D, Sejnowski TJ. Blind separation of auditory event-related brain responses into independent components. Proc Natl Acad Sci USA. 1997;94(20):10979-10984.

32. Jung TP, Makeig S, Westerfield M, Townsend J, Courchesne E, Sejnowski TJ. Analysis and visualization of single-trial event-related potentials. Hum Brain Mapp. 2001(14):166-185.

33. Olbrich S, Jödicke J, Sander C, Himmerich H, Hegerl U. ICA-based muscle artefact correction of EEG data: what is muscle and what is brain? Comment on McMenamin et al. Neuroimage. 2011;54(1):13.

34. Zhang AH, Yang B, Huang L. Feature extraction of EEG signals using power spectral entropy. International Conference on Bio-Medical Engineering and Informatics; Sanya, 2008: pp.435-439.

35. Kim KI, van de Wiel MA. Effects of dependence in high-dimensional multiple testing problems. $B M C$ Bioinformatics. 2008(9):114. 
36. Sarnthein J, Jeanmonod D. High thalamocortical theta coherence in patients with neurogenic pain. Neuroimage. 2008;39(4):1910-1917.

37. Pincus SM, Goldberger AL. Physiological time-series analysis: what does regularity quantify? Am J Physiol. 1994;266(4 Pt 2): H1643-1656.

38. Acharya UR, Fujita H, Sudarshan VK, Bhat S, Koh JEW. Application of entropies for automated diagnosis of epilepsy using EEG signals: A review. Knowl-Based Syst. 2015(88): pp85-96.

39. Wu H, Zhang $\mathrm{H}$, Zheng $\mathrm{C}$, Kong J. Application of power spectral entropy to the noninvasive detection of focal ischemic cerebral injury. Sheng Wu Yi Xue Gong Cheng Xue Za Zhi. 2003;20(2):229-232.

40. Desmedt JE, Tomberg C. Transient phase-locking of $40 \mathrm{~Hz}$ electrical oscillations in prefrontal and parietal human brain reflects the process of conscious somatic perception. Nurosci Lett. 1994;168(12):126-129.

41. Iosifescu DV, Lapidus K. The role of neuroimaging and electrophysiology (EEG) as predictors of treatment response in major depressive disorder. Clin Neuropsychiatry. 2011(8):47-60.

42. Hunter AM, Cook IA, Leuchter AF. The promise of the quantitative electroencephalogram as a predictor of antidepressant treatment outcomes in major depressive disorder. Psychiatr Clin North Am. 2007;30(1):105-124.

43. Graversen $\mathrm{C}$, Olesen $\mathrm{SS}$, Olesen $\mathrm{AE}$, et al. The analgesic effect of pregabalin in patients with chronic pain is reflected by changes in pharmaco-EEG spectral indices. Br J Clin Pharmacol.2012;73(3):363372.

44. Saletu B, Grunberger J, Anderer P, Linzmayer L, Konig P. On the cerebro-protective effects of caroverine, a calcium-channel blocker and antiglutamatergic drug: double-blind, placebo-controlled, EEG mapping and psychometric studies under hypoxia. Br J Clin Pharmacol. 1996;41(2):89-99.

45. Salinsky MC, Binder LM, Oken BS, Storzbach D, Aron CR, Dodrill CB. Effects of gabapentin and carbamazepine on the EEG and cognition in healthy volunteers. Epilepsia. 2002;43(5):482-490.

46. Malver LP, Brokjaer A, Staahl C, Graversen C, Andresen T, Drewes AM. Electroencephalography and analgesics. Br J Clin Pharmacol. 2014;77(1):72-95.

47. Kam JWY, Griffin S, Shen A, et al. Systematic comparison between a wireless EEG system with dry electrodes and a wired EEG system with wet electrodes. Neuroimage. 2018(184):119-129.

\section{Tables}

Table 1 Demographic characteristics of the herpes zoster $(\mathrm{HZ})$ patients in this study. 


\begin{tabular}{|l|l|l|l|l|}
\hline & All & MSP & MRP & $p$-value \\
\hline No. of subjects (male/female) & $80(41 / 39)$ & $45(25 / 20)$ & $25(11 / 14)$ & $0.354^{\mathrm{a}}$ \\
\hline Age, years, mean (SD) & $61.6(12.9)$ & $61.0(11.8)$ & $65.5(8.7)$ & $0.097^{\mathrm{b}}$ \\
\hline Education, years, mean (SD) & $8.8(3.9)$ & $8.9(4.2)$ & $8.5(3.5)$ & $0.956^{\mathrm{c}}$ \\
\hline Duration of illness, days, mean (SD) & $19.8(19.2)$ & $14.6(18.4)$ & $29.2(19.0)$ & $<0.001^{* \mathrm{c}}$ \\
\hline VAS $_{\mathrm{ADM}}$, mean (SD) & $7.2(1.5)$ & $7.1(1.6)$ & $7.6(1.3)$ & $0.149^{\mathrm{c}}$ \\
\hline VAS $_{\mathrm{EEG}}$, mean (SD) & $3.6(1.7)$ & $3.3(1.5)$ & $4.2(1.8)$ & $0.062^{\mathrm{c}}$ \\
\hline VAS $_{\mathrm{DIS}}$, mean (SD) & $4.1(2.3)$ & $2.7(1.4)$ & $6.6(1.4)$ & $<0.001^{* \mathrm{c}}$ \\
\hline
\end{tabular}

Notes: a: Indicates P-values for Pearson Chi-square test

b: Indicates P values for independent two-sample t-test

c: Indicates P-values for independent two-sample Mann-Whitney U test

Table 2 Performances of machine learning models using EEG PSE features to discriminate MSP and MRP patients.

\begin{tabular}{|l|c|c|l|c|}
\hline \multirow{2}{*}{ Predictor } & \multicolumn{2}{|l|}{ Performance, mean \pm SD (\%) } & \multirow{2}{*}{ AUC } \\
\cline { 2 - 4 } & Accuracy & Sensitivity & Specificity & \\
\hline LDA & $71.4 \% \pm 7.1 \%$ & $88.2 \% \pm 12.3 \%$ & $34.0 \% \pm 25.9 \%$ & 0.67 \\
\hline k-NN & $80.0 \% \pm 11.7 \%$ & $82.5 \% \pm 14.7 \%$ & $77.7 \% \pm 27.3 \%$ & $\mathbf{0 . 8 5}$ \\
\hline SVM & $77.1 \% \pm 6.0 \%$ & $88.2 \% \pm 12.1 \%$ & $55.6 \% \pm 13.0 \%$ & 0.76 \\
\hline RF & $67.1 \% \pm 6.4 \%$ & $81.6 \% \pm 11.2 \%$ & $42.7 \% \pm 23.0 \%$ & 0.59 \\
\hline VAS $_{\text {EEG }}{ }^{*}$ & $58.6 \%$ & $69.1 \%$ & $42.9 \%$ & N/A \\
\hline
\end{tabular}

Notes: * For comparison, we also used $\mathrm{VAS}_{\mathrm{EEG}}$ (i.e., the pain ratings measured right before $\mathrm{EEG}$ acquisition) to predict the medication effects, and the criteria was, if a patient's VAS $\mathrm{EEG}_{\mathrm{E}}$ was less than half of VAS $\mathrm{ADM}_{\mathrm{A}}$ or VAS $\mathrm{E}_{\mathrm{EEG}}$ was less than 3 , the patient was defined as MSP.

Abbreviations: LDA: Linear Discriminant Analysis; k-NN: k-nearest neighbors with k=5; SVM: Support Vector Machine; RF: Random Forest; AUC: Area Under ROC Curve.

\section{Figures}



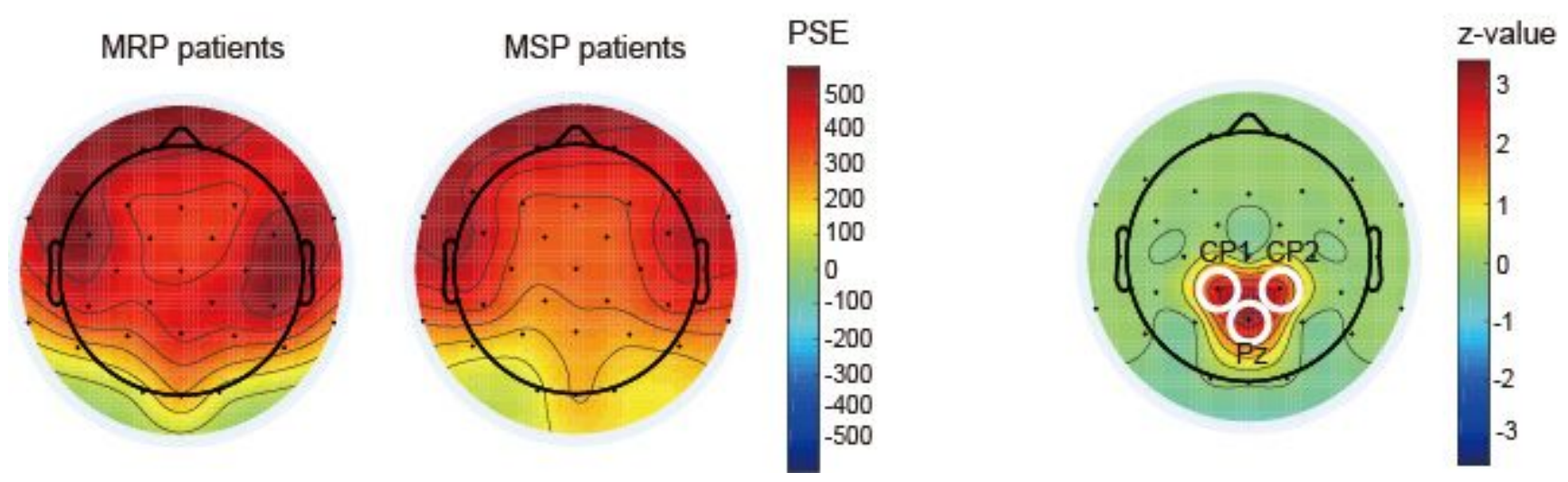

Figure 1

Comparison of PSE between MSP and MRP patients. Channels with significant PSE differences (CP1, $\mathrm{CP} 2, \mathrm{Pz})$ are indicated by white circles ( $<<0.05$, FDR-corrected). The topographical distribution of $z$-value (two-sample rank sum test) in the beta band is shown in the right.

\section{MRP patients $\square$ MSP patients}

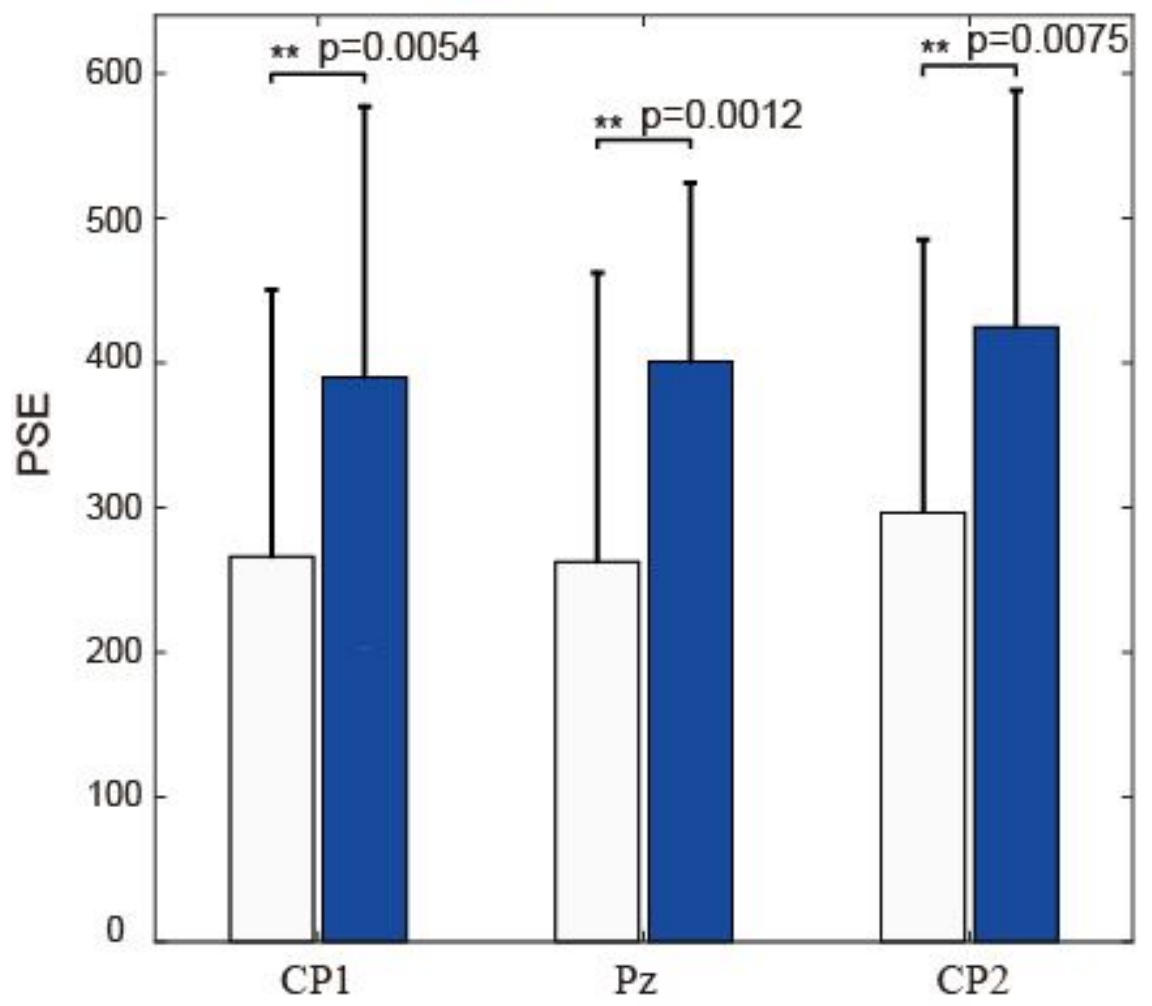

Figure 2

Comparison of PSE at CP1, CP2 and Pz in beta band between MSP and MRP patients. 


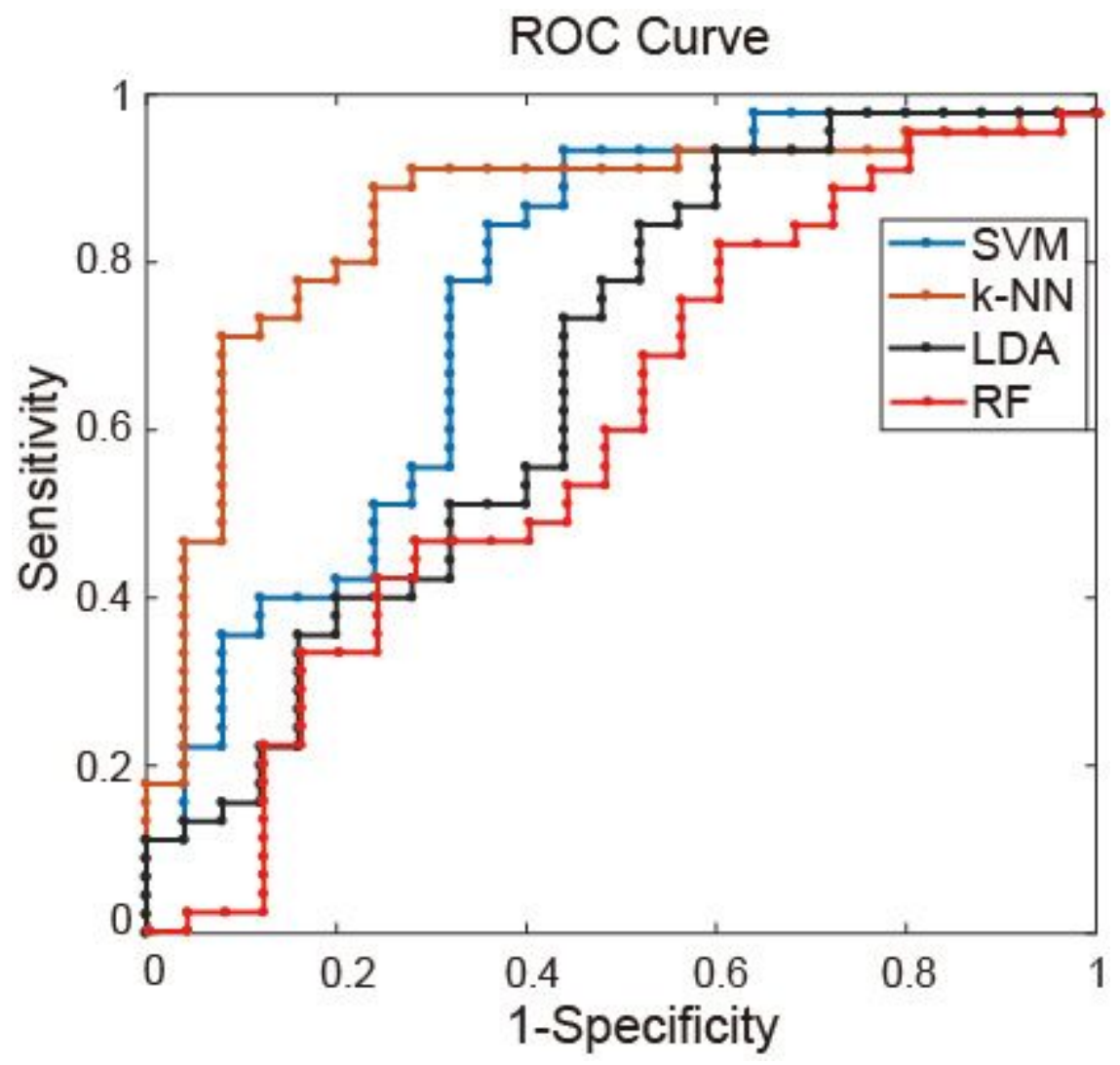

Figure 3

Receiver Operating Characteristic (ROC) curves of four classifiers. 